\title{
DIGITAL IMAGE PROCESSING IN THE ANALYSIS OF ASTROMETRIC PLATES.
}

\author{
A. LOPEZ GARCIA ${ }^{1}$, J. L. VALDES ${ }^{1}$, J. A. LOPEZ ORTI ${ }^{1,2}$ \\ R. LOPEZ. MACHI ${ }^{1,2}$, G. J. PEREZ ${ }^{1}$. \\ ${ }_{1}^{1}$ Observatorio Astronómico de la Universidad de Valencia. (OAUV) \\ ${ }^{2}$ Colegio Universitario de Castellón. \\ ${ }^{1}$ Departamento de Matemática Aplicada y Astronomía. \\ Universidad de Valencia. SPAIN.
}

ABSTRACT. In this paper, we display an improvement to our process of semi-automatic measuring of astrometric plates, in which the photometric sensor is substituted by a CCD system of image getting and digitalization. The advantages of this method are analyzed taking into account the possibilities of the image analysis in the space and frequency domain.

\section{Introduction}

In the Astronomical Observatory of Valencia we have a program of selected minor planets observations since 1984. To this aim we have developed both the photographic equipment and the mechanical and electronic devices for plate measuring as well as the software support to whole process. The optical system includes an ocular and photoelectric photometer heading.

Once two stars (E1 and E2) have been selected, the several expositions observed and the sequence of the objects to be measured defined, the process of measuring in each position of the plate-holder is automatic. The process is repeated after a tum of $180^{\circ}$ of the plate-holder.

An automated position measure is applied for each star and for the minor planet. It includes reading local plate background transparency, an approximate and fine centering and the automatic measuring process of each image.

As the separation of the two or three expositions of the objects is about 0.3 to $1 \mathrm{~mm}$, all the images cannot be seen in the ocular field ( $1 \mathrm{~mm}$ wide) at the same time, making tedious star finding and centering, and easy to get in troubles in the full process.

Details of the measure process are shown in (Lopez, A. et al.: 1988), (Lopez, A. et al.: 1990) and (Lopez, A. et al.: 1989a).

In this paper we present the new process of image detection and identification, giving better results and being easier, quicker and more robust than the previous one with the photometer.

\section{2.- CCD Image detection}

An alternate centering process reading all the pixels around the image with a two dimensional sensor (CCD camera plus digitizer board) and getting the center of transmitted light has given better results, as shown in (Lopez, A. et al.: 1989b). 
From that, several changes have been applied in order to improve the plate measuring process.

The binocular reading head has been substituted by a small CCD camera and a digitizer board on the computer, so that the field containing the several exposures can be seen on the screen, the pixels in a window around the images read and the image positions detected.

The plate measuring process begins with the centering of the two initial stars on the screen (3x4 mm across) (Fig. 1).

The scale and tilt of the CCD camera versus the microscope $X, Y$ screws are obtained by making movements of the first image of $\mathrm{E} 1$ and determining its position over four cross small windows.

The expositions of E1 and E2 are measured in a small central window and the relative position of the images is determined for every object to be measured.

The sequence of stars and asteroid is defined and the measure process begins.

\section{3.- Digital image analysis}

For each object (star and asteroid) the detection process is global, including all the expositions at the same time.

Our purpose is to get positions of all images relative to the camera field center in a way as fast and sure as possible working in a real time process. So, applied steps are simple and efficient.

A central window is defined including all the images of the object (Fig. 2). As we measure the plates in two opposite positions, the window is elongated in the horizontal direction of screen, as the several images of the object appear.

The window is divided into $10 \times 6$ squares with ten by ten pixels each, and the images are identified by the signal detected in these squares. As the size of the squares is similar to the images diameters, each image will give positive signal at least in one or two adjacent squares, side by side or in diagonal. Other spurious marks of the plate will active squares isolated or in irregular chains (Fig. 3).

The process of identifying and measuring the stellar images is the following:

1.- The maximum and minimum pixel signal inside the window described above are determined.

2.- In each square the pixels with signal below a threshold give, when added, the square signal value. The greatest value is needed to select the active squares. chained.

3.-The connectivity of active squares is determined, classifying them into isolated and

4.- For the chained squares, an analysis is made and the members of each group are determined. center.

5.- All the active groups are measured again, determining the $\mathrm{X}, \mathrm{Y}$ coordinates of its light

6.- A small square window is defined around this center and the final $X, Y$ position determined. Some other parameters can be obtained if necessary (brightness, shape, orientation, etc) (Fig. 4).

7.- The identification of active groups with object images is the final step of this process. For that, we compare the $\mathrm{X}$ and $\mathrm{Y}$ distances of the several expositions with the theoretical values calculated from E1 and E2 (Fig. 5). 


\section{4.- Future improvements}

We will also apply other routines to special problems involved in our minor planets work, specially when the asteroid image is very weak. Masks and Fourier analysis techniques will be investigated and applied in this context in the next future.

The use of masks in the previous analysis of images is extended and digitizer boards hardware and software have many possibilities. Nevertheless the use of masks is dangerous as it modifies the real structure of signals.

It will be possible to identify the object images structure in the frequency domain comparing its principal components with the Fourier coefficients of the theoretical positions, size and shape of the object images, although the measure process time will increase substantially (Gonzalez, R.C.; Wintz, P., 1987).

\section{5.- Conclusions}

This method of identifying and measuring image positions in the context of astrometric plates, improves substantially the results of our previous photometric measuring process.

It can by extended to other similar problems such as the measure of PZT plates and astronomical plates in general.

Major astronomical image processing systems (MIDAS, IRAF, INVENTORY, COSMOS, etc) include many commands that can perform the analysis described here (Murtagh, F.: 1989).

Nevertheless, we think it is better in our work to develop a special software, that will be improved in the next future, in order to extend the confidence of the full process of measurement of astrometric plates.

\section{References}

Gonzalez, R.C.; Wintz, P.: 1987, Digital Image Processing, Adisson-Wesley.

Lopez , A. et al.: 1988, Boletín Astronómico del Observatorio de Madrid. Vol.XII, 1, p. 1.

Lopez , A. et al.: 1989a, XI EUROPEAN REGIONAL ASTRONOMY MEETING OF THE IAU (La Laguna).

Lopez , A. et al.: 1989b, ERRORS, BIAS AND UNCERTAINTIES IN ASTRONOMY. Strasbourg.

Lopez, A. et al.: 1990, ASTEROIDS, COMETS AND METEORS III Meeting

Uppsala Universitet, p. 125.

Murtagh, F.: 1989, Data Analysis in Astronomy III, Plenum Press, p. 111. 


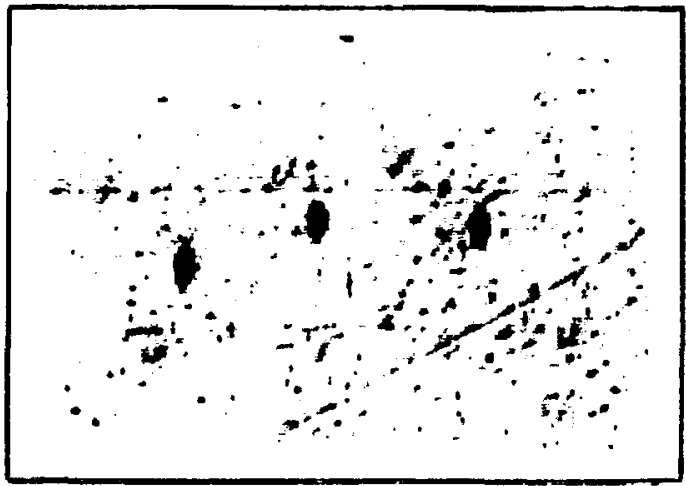

Fig. 1.- Visible plate field in the CCD camera

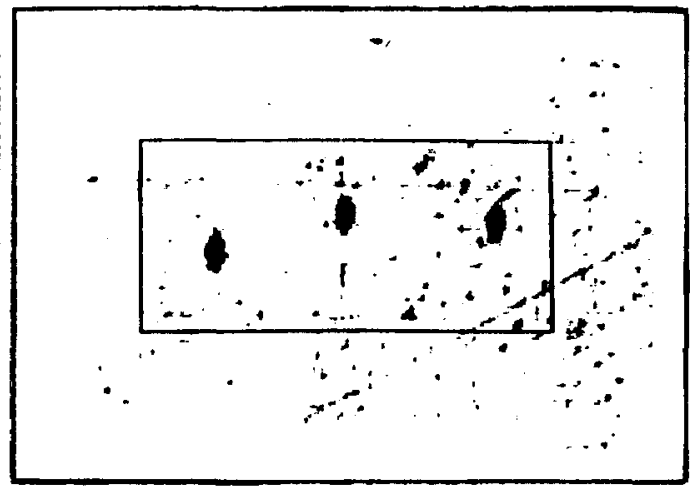

Fig. 2.- Central window where the analysis is performed.

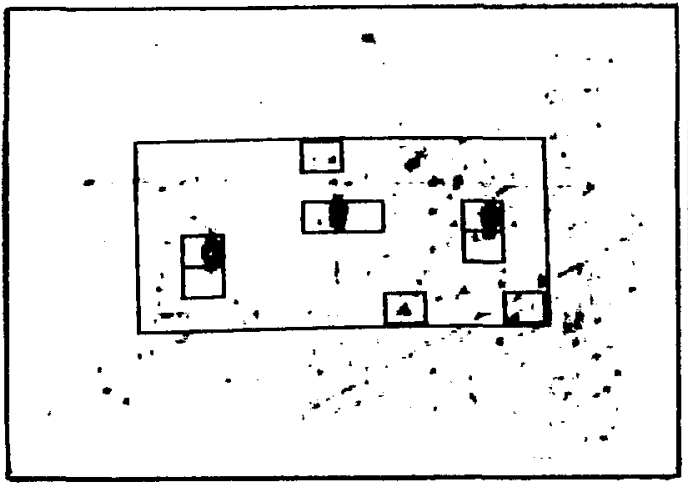

Fig. 3.- Squares with active transmitted light signal.

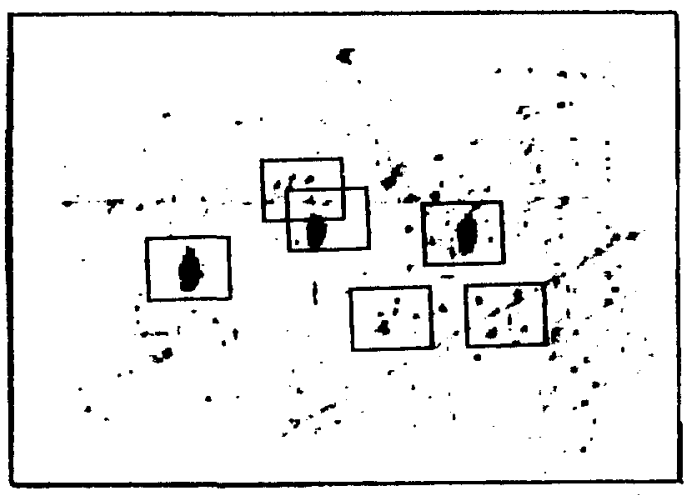

Fig. 4.- Result of the active groups re-centering process.

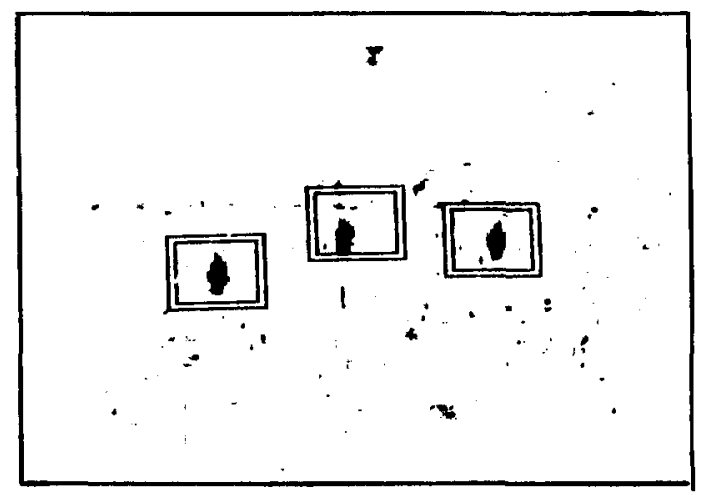

Fig. 5.- Object images finally identified. 